\title{
El origen de la desigualdad y la progresividad en la distribución de la financiación de las Comunidades Autónomas de régimen común
}

\author{
Luis Ángel Hierro Recio \\ Pedro Atienza Montero \\ Rosario Gómez-Álvarez Díaz \\ Departamento de Historia e Instituciones Económicas y Economía Política \\ Facultad de Ciencias Económicas y Empresariales \\ Universidad de Sevilla \\ Av. Ramón y Cajal, 1 \\ 41018 Sevilla
}

\begin{abstract}
RESUMEN
El presente trabajo tiene por objetivo analizar la contribución de las distintas fuentes de ingresos de las Comunidades Autónomas de régimen común a la desigualdad y la progresividad del sistema de financiación autonómica en su conjunto. En el apartado 1 se resume la literatura sobre medición de los desequilibrios financieros horizontales en sistemas de financiación federales, constatándose la ausencia de instrumentos que midan el impacto individual de cada fuente de ingresos. En el apartado 2 se propone la metodología a aplicar, en concreto la descomposición natural de los índices de concentración y la descomposición directa del índice de Suits. En el apartado 3 se presentan los resultados de la aplicación de la metodología propuesta. Y finalmente en el apartado 4 se presentan las conclusiones, resultando que es el Fondo de Suficiencia,
\end{abstract}


en cuanto variable cierre del sistema, el que determina la distribución final de recursos y su sentido progresivo, pero que al perderse una parte de progresividad por el comportamiento de los tributos cedidos resulta imprescindible el FCI, para compensar dicha pérdida.

Palabras clave: Financiación autonómica, federalismo fiscal, transferencias intergubernamentales, desigualdad, progresividad.

Clasificación JEL: H71, H73.

\begin{abstract}
The present paper has as aim to analyse the contribution to the inequality and progressiveness of financing resources of Comunidades Autónomas. In the first part we resume the literature on measurement of the horizontal fiscal imbalances in systems of federal funding, where we confirm the absence of instruments that measure the individual impact of each source of revenue. In the part 2 we propose the methodology to apply, in concrete the natural decomposition of the indexes of concentration and the direct decomposition of the index of Suits. In the part 3 we present the results of the application of the methodology proposed. And finally in the part 4 we present the conclusions, resulting that it is the Fondo de Suficiencia, as variable closing of the system, who determines the final distribution of resources and his progressive sense, but since a part of progressiveness disappear by the behaviour of the tributos cedidos, results indispensable the FCI to compensate said loss.
\end{abstract}

Keywords: Fiscal federalism, intergovernmental grants, inequality, progressiveness.

JEL Classification: H71, H73.

\title{
1. INTRODUCCIÓN
}

Una de las cuestiones en materia de federalismo fiscal que recientemente está recibiendo una atención antes no prestada es la referida a la medición del impacto redistributivo o nivelador de las transferencias intergubernamentales. De hecho, hasta muy recientemente los estudios que pretendían realizar este tipo de mediciones eran inexistentes y hay que remontarse poco en el tiempo para encontrar trabajos dedicados a esta 
tarea. Por lo general, todos ellos tienen por objetivo medir el impacto nivelador que se produce cuando a los ingresos que obtienen por tributos los Estados en países federales se les agregan las transferencias. Así, en Martínez Vázquez, Boex (1999) se utiliza el coeficiente de variación para medir la nivelación de los desequilibrios financieros horizontales en Rusia para el período 1992-1997. En el mismo se cuantifica la dispersión de las fuentes de ingreso mediante un sistema acumulativo: en primer lugar los recursos tributarios, tanto propios como compartidos, más tarde la transferencia de nivelación ${ }^{1}$ y finalmente las restantes transferencias condicionadas. Las conclusiones de dicho análisis son que la transferencia de nivelación produce una reducción del coeficiente de variación del 1,39 para el total de ingresos tributarios al 1,22 una vez que se acumula dicha transferencia. Finalmente, cuando se toma el resto de transferencias y el conjunto de ingresos financieros (excluida la deuda pública) el coeficiente pasa a tener un valor de 1,18. Esta misma metodología se aplica en Brodjonegoro y Martínez Vázquez (2002) para el caso de Indonesia, utilizando tanto el coeficiente de variación como la desviación típica como indicadores de los desequilibrios financieros horizontales, obteniéndose como resultado que al añadir los tributos compartidos a los tributos propios ambos índices aumentan ligeramente, y que, cuando se añade la transferencia de nivelación, el coeficiente de variación se reduce drásticamente, mientras que la desviación típica aumenta muy ligeramente.

Un sistema similar es el que se sigue en Hierro, Atienza y Patiño (2007a y 2007b). En el primero, dedicado al estudio de los casos de Alemania, Australia, Canadá, España y Suiza, se utilizan, además de los indicadores citados más arriba, índices de concentración antes y después de transferencias y sus correspondientes curvas de concentración para estudiar las variaciones en la distribución de los recursos. Los resultados que se obtienen son que en España es donde mayor igualación se produce tras las transferencias, siendo sólo comparable en este sentido con Alemania, y también donde se obtiene una distribución final de recursos más igualitaria después de transferencias junto a Australia. Por su parte, el segundo de los trabajos se centra en Australia para intentar determinar los efectos no ya del conjunto de las transferencias sino de cada una de ellas. Para ello lo que hace es seguir un método secuencial similar al utilizado en Martínez Vázquez, Boex (1999), aunque en este caso utilizando índi-

${ }^{1}$ El denominado Fondo para el Apoyo Financiero de las Regiones (FFSR, sus siglas en inglés). 
ces de concentración como en el estudio anterior y, además, mediante una adaptación de la metodología de Aronson, Johnson y Lambert (1994) de manera que se descompone el efecto redistributivo de cada tipo de transferencia en un impacto de igualación, otro de no igualación y, por último, de reordenación. La secuencia acumulativa de recursos que se sigue es la siguiente: recursos tributarios, transferencias condicionadas sujetas a nivelación, transferencia de nivelación y, por último, transferencias condicionadas no sujetas a nivelación, y la conclusión que se obtiene es que son las transferencias condicionadas sujetas a nivelación las causantes de la mayor parte de la reducción de la desigualdad, seguida de la transferencia de nivelación cuya fuerte reordenación le hace perder impacto redistributivo.

Ahora bien, estos estudios no sólo pretenden conocer el impacto redistributivo de las transferencias sino que además intentan analizar el sentido de la redistribución. Para ello Horman, Cordeira (2004), en un estudio referido a China, Tailandia, Vietnam, Indonesia y Filipinas, utilizan la elasticidad renta de los ingresos y gastos subcentrales para calificar si el sistema es o no nivelador. Para ello regresionan los logaritmos de los ingresos y de los gastos en términos per cápita con el logaritmo de la renta per cápita subcentral, de forma que si la elasticidad renta de los gastos es mayor a la de los ingresos se produce un resultado nivelador. Así ocurre en Indonesia, China y Vietnam donde son sensiblemente mayores las elasticidades renta de los gastos que las de los ingresos. Con un enfoque muy similar, Rao (2000) estima la nivelación a partir del cálculo de la elasticidad renta de las transferencias mediante un análisis de regresión en un modelo log-lineal para el caso de la India; en este caso ya no se comparan gastos e ingresos sino que lo que se hace es tomar directamente las transferencias y ver cómo evolucionan en relación con la renta mediante el concepto de elasticidad.

De otra parte, en los dos trabajos de Hierro, Atienza y Patiño (2007a y 2007b) se utilizan variaciones de los índices de Kakwani y Suits para medir la progresividad o regresividad de la redistribución asociada a las transferencias. En su versión original ${ }^{2}$, el índice de Kakwani se calcula a partir de la diferencia de los índices de concentración de la curva de concentración de los impuestos considerados y la curva Lorenz típica representativa de la renta antes de impuestos; y el índice de Suits como un índice de concentración, pero en este caso de la curva de concentración relativa de los impuestos, que en ordenadas sitúa la acumulación porcentual de los impuestos y en abcisas

\footnotetext{
${ }^{2}$ Véase Kakwani (1977) y Suits (1977).
} 
la acumulación porcentual de la renta. La adaptación realizada en estos trabajos consiste en calcular los mismos índices pero referidos, el primero, a la curva de concentración de los ingresos de los Estados y la curva de Lorenz del PIB (o renta) de los Estados y, el segundo, a la curva de concentración relativa de los ingresos. Además, en el segundo de ellos, el referido a Australia, se utiliza el mismo método ya explicado de agregación sucesiva de los distintos tipos de transferencias para descomponer el impacto, en este caso de aumento de la progresividad, obteniendo como conclusión que la progresividad final de la distribución de recursos tiene su origen en la transferencia de nivelación.

Como se ha podido observar, cuando se pretende analizar el aporte de cada instrumento de financiación a la distribución de recursos la práctica totalidad de los trabajos lo que hace es agregar la correspondiente fuente de ingresos y calcular la medida de desigualdad utilizada para obtener la diferencia con respecto a la situación previa a añadir dicha fuente. Esta metodología es adecuada para medir la variación en la igualdad derivada de la incorporación de un nuevo instrumento de financiación, en su caso las transferencias en su conjunto, o para medir el impacto de una variación en las características de instrumentos ya existentes. Ahora bien, cuando lo que pretendemos es valorar el aporte que realiza cada uno de los instrumentos de financiación a la distribución final, el proceso secuencial de ir añadiendo fuentes de ingreso hace que el valor del indicador para cada fuente dependa del orden en que se añadan las mismas. Dicho de otra forma, el impacto distributivo por ejemplo del Fondo de Compensación Interterritorial en la financiación de las CC.AA. no será el mismo si lo calculamos tomando como punto de partida los ingresos por tributos que si tomamos como ingresos iniciales los tributos y el Fondo de Suficiencia. Siendo así, el impacto redistributivo dependería del juicio de valor por el que se ordenasen las fuentes de ingresos y sólo si existe un acuerdo generalizado del orden seguido éste podrá ser considerado una medición objetiva del impacto redistributivo. Ese puede ser el caso por ejemplo de la nivelación en Alemania donde el sistema de transferencias está definido en un orden secuencial o el de los ingresos del Fondo de Suficiencia con respecto a los tributos cedidos en España. Sin embargo, difícilmente sería aceptable, por ejemplo, que intentásemos establecer un orden único entre los propios tributos cedidos para conocer el impacto de cada uno de ellos. Es más, en el caso de España existen recursos como los procedentes del Fondo de Compensación Interterritorial que no son ordenables con respecto a los restantes ya que para las CC.AA. que los reciben tienen el mismo nivel de protección constitucional que los tributos cedidos o los tributos propios, sin que mantengan una relación de dependencia con éstos o con cualquier otra transferencia.

Pues bien, la ausencia de un orden objetivo de la totalidad de los recursos que 
obtienen las CC.AA. hace necesario acudir a otros instrumentos de medida tanto de la desigualdad en la distribución de recursos como de su sentido, lo cual constituye precisamente el objetivo que nos fijamos para el presente trabajo. Así, en el siguiente apartado se describe dicho objetivo y la metodología empleada, en el apartado tres se presentan y se comentan los resultados, y finalmente se concluye con un apartado de conclusiones.

\section{2. ОвJеtivo y мÉtodo}

En efecto, si el establecimiento de órdenes secuenciales en los recursos de las CC.AA. incorpora juicios de valor, es necesario plantear medidas alternativas que permitan evaluar la aportación de cada fuente a la desigualdad en la distribución total y si es posible encontrar la forma de valorar la aportación individual a la progresividad de la misma. En realidad, tal como se plantea la cuestión nos encontramos ante un problema de descomposición aditiva de índices de desigualdad, problema éste muy tratado por la literatura relativa al estudio de la distribución de la renta y que, teniendo en cuenta los estudios precedentes, podemos resolver satisfactoriamente ${ }^{3}$.

Entre los indicadores de desigualdad más simples que permiten su descomposición aditiva por factores se encuentra el índice de Concentración ${ }^{4}$, que además de ser independiente del nivel medio de la variable y de la población (Homogeneidad de grado cero) cumple el principio Pigou-Dalton ${ }^{5}$. Su descomposición presenta cierta dificultad ya que el orden en el que se acumula la población, en nuestro caso las CC.AA., y por ende los ingresos, incide en el valor del índice. En efecto, siendo $C(x)$ el índice de Concentración de los recursos per cápita provenientes del total de financiación,

${ }^{3}$ Para un repaso de la literatura sobre la cuestión ver Rao (1969), Fei, Ranis y Kuo (1978), Lerman, Yitzhaki (1985) o Shorrocks (1982, 1999).

${ }^{4}$ Otros indicadores de desigualdad descomponibles aditivamente por factores son la varianza y el cuadrado del coeficiente de variación.

${ }^{5}$ Este principio nos dice que cualquier transferencia desde una unidad rica a otra pobre, en nuestro caso una transferencia desde una C.A. con mayor volumen de recursos de financiación per cápita hacia otra con menor volumen y que no invierta sus órdenes relativos (que la C.A. que antes de la transferencia tenía mayor volumen per cápita no pase a poseer menos que la otra), debe dar como resultado que el índice exprese una disminución de la desigualdad. 
$C(x k)$ el índice de Concentración de los recursos per cápita provenientes del instrumento de financiación $k, T k$ el volumen total de recursos del instrumento $k$ e $T$ el fondo global de la financiación total se puede demostrar que ${ }^{6}$ :

$$
C(x) \leq \sum_{k} \frac{T k}{T} C\left(x_{k}\right)
$$

Dicha desigualdad se produce porque la distribución de cada fuente singular de ingresos determina una ordenación de las CC.AA., ordenación ésta que no tiene por qué coincidir con la del total de recursos. Ese problema se resuelve mediante lo que Shorrocks denomina la «descomposición natural» del índice ${ }^{7}$, cuya expresión aplicada a nuestro caso sería:

$$
C(x)=\sum_{k} \frac{T k}{T} \bar{C}\left(x_{k}\right)
$$

Donde $C\left(x_{k}\right)$ representa el pseudo-índice de Concentración de los recursos per cápita provenientes del instrumento de financiación $k$ manteniendo el orden de las CC.AA. que genera la financiación total en lugar del orden que genera la propia fuente.

Es decir, el índice de Concentración global se descompone como la suma ponderada de los pseudos-índices de Concentración de cada recurso de financiación, siendo el factor de ponderación el porcentaje que representan el ingreso de cada fuente en relación al total de ingresos obtenidos.

La aplicación que se acaba de describir permite descomponer la desigualdad desde una perspectiva cross-section y, en consecuencia, medir en qué parte la desigualdad total es fruto de cada instrumento de financiación. Ahora bien, el cálculo del índice de Concentración y de su descomposición aditiva no nos posibilita calificar la desigualdad. Así, podemos conocer que una determinada subvención aporta más o menos a la desigualdad final pero no si la misma está orientada en el sentido de favorecer en su

\footnotetext{
${ }^{6}$ Véase Fei, Ranis y Kuo (1978) o Shorrocks (1982).

${ }^{7}$ Previamente ya demostrada por Rao (1969) o Fei, Ranis y Kuo (1978).
} 
distribución a las CC.AA. con menor capacidad fiscal o viceversa. Para ello debemos dar un paso más utilizando alguno de los índices más arriba citados. En concreto podemos utilizar el índice de Suits para determinar en qué sentido se distribuye cada fuente de ingresos. La ventaja de la descomposición del índice de Suits es que su descomposición aditiva no implica el uso de pseudos-índices ni requiere de ninguna «descomposición natural» ya que para el mismo, como se ha explicado, la curva de concentración se obtiene ordenando siempre en función de la renta (o PIB). Es decir, el índice de Suits de la financiación total es estrictamente igual a la suma ponderada de los índices de cada uno de los instrumentos que la componen.

En efecto, si denominamos $R k(y)$ la función representativa de la curva de concentración relativa del recurso $k$, que indica el porcentaje de ingresos acumulado procedentes de la fuente financiera $k(k=1, \ldots r)$, por las CC.AA. que acumulan el $y$ por ciento de la renta total del país, expresado en tanto por uno, la cantidad de ingresos del tipo $k$ recibida por las CC.AA. que acumulan el $y$ por ciento de la renta vendrá dada por:

$$
Z_{k}(y)=T_{k} \cdot R_{k}(y)
$$

Y para el conjunto de los ingresos por:

$$
Z(y)=\sum_{k} T_{k} \cdot R_{k}(y)
$$

Siendo $T_{k}$ y $T$ respectivamente las cantidades totales del recurso $k$ y del conjunto de recursos recibidas por las CC.AA., tenemos que $T=\sum_{k} T k$

El porcentaje total de recursos acumulados por las mismas que representan el $y$ por ciento de la renta vendrá dado por:

$$
\sum R(y)=\frac{Z(y)}{T}=\frac{\sum_{k} T_{k} \bullet R_{k}(y)}{T}
$$

Dado que el índice de Suits para el conjunto de los ingresos se expresa como: 


$$
S=1-2 \int_{0}^{1} R(y) d y
$$

Sustituyendo tenemos que:

$$
S=1-2 \int_{0}^{1} \frac{\sum_{k} T_{k} \bullet R_{k}(y) d y}{T}=1-T \frac{2}{T} \int_{0}^{1} \sum_{k} T_{k} \bullet R_{k}(y) d y
$$

Operando tenemos que:

$$
S=\frac{\sum_{k} T_{k}-2 \int_{0}^{1} \sum_{k} T_{k} \bullet R_{k}(y) d y}{T}=\frac{\sum_{k} T_{k}\left(1-2 \int_{0}^{1} R_{k}(y) d y\right)}{T}
$$

Es decir:

$$
S=\sum \frac{T_{k}}{T} S_{k}
$$

En resumen, los índices de Suits de cada uno de las fuentes de ingreso así como del total de financiación se calculan manteniendo una misma ordenación de las CC.AA., la ordenación proveniente de los PIB per cápita de cada una de las mismas, lo que determina que la descomposición aditiva del índice de Suits sea directa. Esto supone que además de poder determinar la contribución de cada recurso a la desigualdad total resultante en cada período, estamos en condiciones de poder valorar cuánto aporta a la progresividad o regresividad cada uno de los instrumentos.

\section{Los REsultados}

En la Figura 1 se representa de forma simplificada la estructura del sistema de financiación de las CC.AA. de régimen común y en los Cuadros 1 y 2 aparecen la distribución territorial de la totalidad de las fuentes de financiación, en miles de euros y en euros per cápita respectivamente. Posteriormente, tomando las cifras que aparecen 
en dichos cuadros se ha estimado la contribución de cada recurso de financiación tanto a la desigualdad como a la progresividad globales del conjunto del sistema de financiación autonómica. Los resultados aparecen en el Cuadro 3.

Figura 1. Estructura del sistema de financiación de las CC.AA. de régimen común

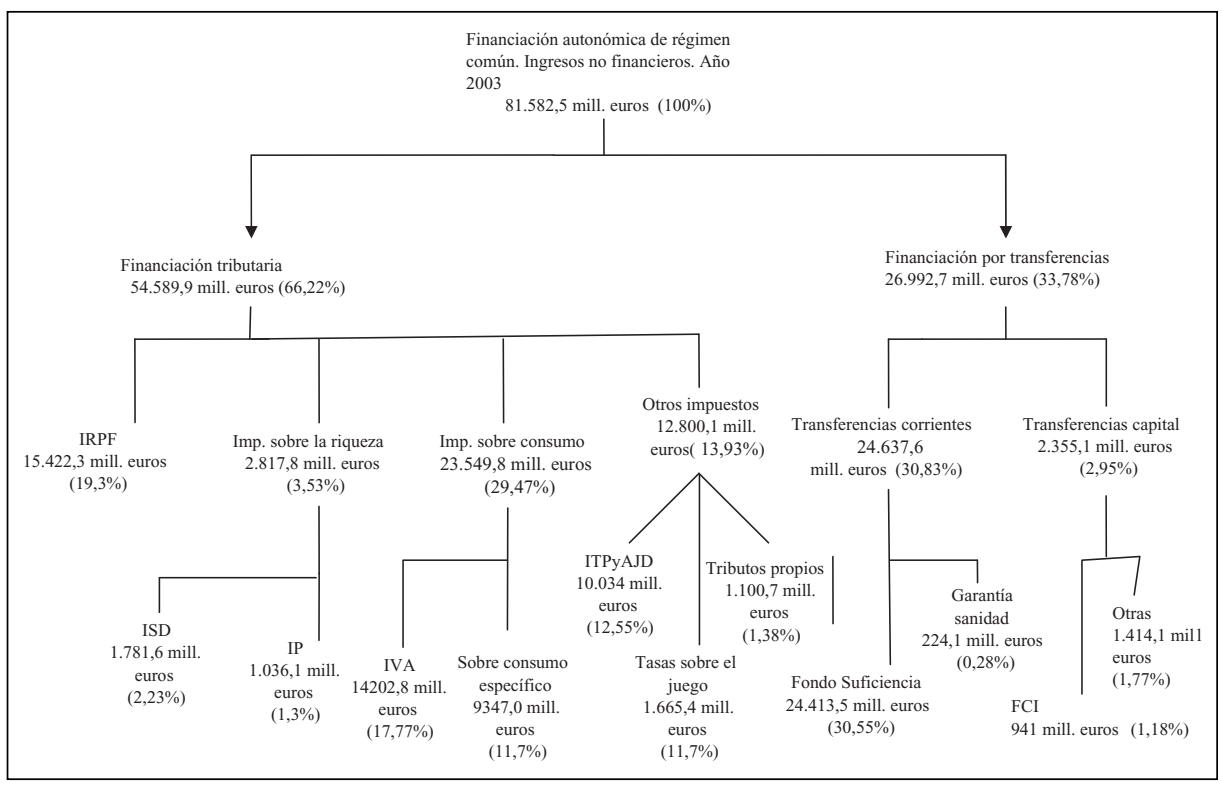

Fuente: Elaboración propia a partir del Cuadro 1.

Para una interpretación adecuada de los resultados obtenidos en el Cuadro 3 es necesaria una referencia inicial a la posibilidad de que los índices tomen valor negativo. En efecto, refiriéndonos primero a los pseudos-índices de concentración utilizados para medir la parte de desigualdad debida a una determinada fuente, si aparece un valor con signo negativo está indicando que la fuente en cuestión produce una desigualdad que opera en contra de la desigualdad final o, lo que es lo mismo, que si se ordenan las CC.AA. siguiendo el orden procedente de esa fuente de ingresos el resultado es una ordenación inversa a la que se obtiene considerando el total de los ingresos. Cuanto menor sea el valor del pseudo-índice de concentración negativo más inversa es la distribución de la fuente con respecto a la resultante del conjunto de ingresos. Por su parte, en cuanto al valor negativo en el índice de Suits lo que indica es que el resultado de la distribución favorece a las CC.AA. con menor PIB, es decir, indica progresividad en la distribución. 


\begin{tabular}{|c|c|c|c|c|c|c|c|c|c|c|c|c|c|c|c|c|c|c|c|c|}
\hline & & 홍 & 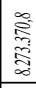 & है & 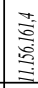 & 路 & 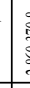 & & 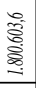 & 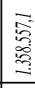 & 高 & 沀 & 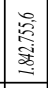 & 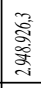 & 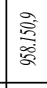 & $\begin{array}{l}\text { ळे } \\
\approx \\
\text { ळे }\end{array}$ & 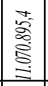 & 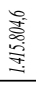 & 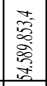 & \\
\hline & & 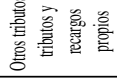 & 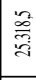 & $\begin{array}{ll}\stackrel{1}{\infty} \\
\stackrel{\infty}{\rightleftharpoons}\end{array}$ & 管 & & & & 8 & 点 & 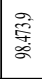 & 蒿 & 产 & 8 & 总 & $\underset{\substack{\infty \\
\infty}}{\infty}$ & 总 & 兴 & 然 & \\
\hline & & 烝总总 & 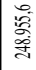 & Ẽ & 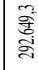 & 8 & & & 䒽 & 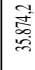 & Fे & 8 & 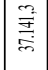 & 产 & 亳 & $\begin{array}{l}\frac{2}{5} \\
\stackrel{5}{3}\end{array}$ & $\mid$ & $\begin{array}{c}\infty \\
\vdots \\
\vdots \\
\vdots \\
\vdots\end{array}$ & $\mid$ & $\frac{1}{y_{y}}$ \\
\hline & & 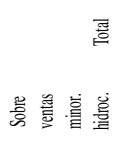 & 等 & $\begin{array}{l}\text { 商 } \\
\text { ơ } \\
8 \\
8\end{array}$ & 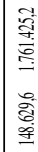 & 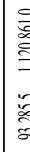 & : & 是 & 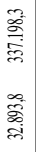 & 产 & 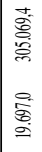 & 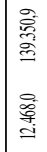 & 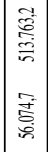 & 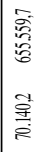 & 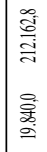 & 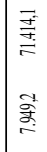 & 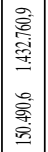 & 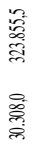 & 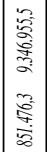 & 紫 \\
\hline & & 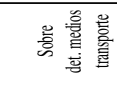 & हैं & 8 & 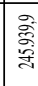 & & & t: & 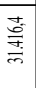 & Eे & 高 & 言 & 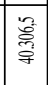 & $\overrightarrow{\bar{A}}$ & 尊 & 总 & 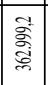 & 商 & 角 & 5 \\
\hline & & 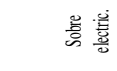 & 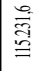 & 高 & 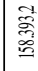 & 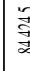 & & & 产. & 离 & 舞 & 善 & 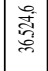 & $\vec{~}$ & 袁 & สิ่ & 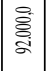 & ळे & 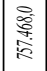 & 戀 \\
\hline & 谤 & 总童兽 & 趈 & $\therefore$ & 䓂 & 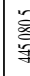 & 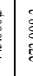 & 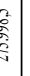 & $\begin{array}{l}\overrightarrow{0} \\
\text { o. } \\
\underline{\underline{a}}\end{array}$ & 居 & 要 & $\frac{2}{3}$ & 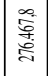 & \begin{tabular}{|l} 
总 \\
害 \\
\end{tabular} & 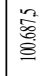 & 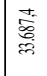 & $\mid$ & 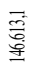 & 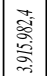 & 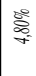 \\
\hline & 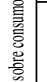 & 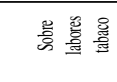 & 离 & 8 & $\begin{array}{l}\frac{2}{5} \\
\stackrel{5}{6}\end{array}$ & 㱐 & & 害 & $\begin{array}{l}3 \\
3 \\
3 \\
3 \\
3\end{array}$ & 商 & 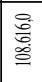 & 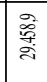 & \begin{tabular}{|l|}
2 \\
言 \\
8
\end{tabular} & 高 & 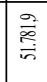 & 党 & 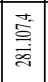 & हू & 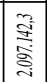 & 想 \\
\hline & 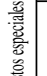 & 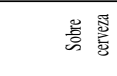 & 管 & 管 & : & 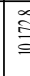 & & 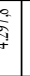 & 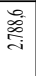 & สี่ & 今े & 童 & 空 & 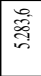 & 尊 & 음 & 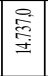 & ถิ & \begin{tabular}{|c|}
\multirow{3}{*}{} \\
aे
\end{tabular} & $\frac{50}{3}$ \\
\hline 褰 & 产 & 总总言 & $\stackrel{\widetilde{\circ}}{=}$ & 察 & 察 & : & & 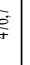 & 落 & है & 这. & 䄈 & 范 & 墥 & 童 & is & $\stackrel{2}{\Xi}$ & बे & 总 & $\frac{5}{8}$ \\
\hline & & 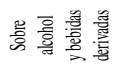 & 离 & 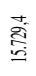 & 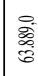 & 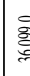 & 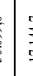 & 尃 & $\begin{array}{l}\text { 总 } \\
= \\
=\end{array}$ & के & 言 & 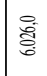 & 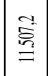 & 递 & 言 & 蕞 & $\mid$ & 祭 & 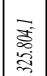 & $\frac{5}{3}$ \\
\hline & & 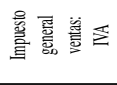 & s. & 8 & 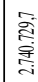 & 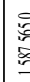 & & E. & $\begin{array}{l}\text { 足 } \\
\frac{8}{7} \\
\frac{7}{y}\end{array}$ & 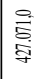 & 究 & 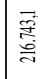 & 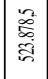 & 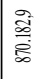 & 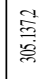 & $\begin{array}{l}\text { 悉 } \\
\stackrel{g}{g}\end{array}$ & 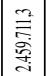 & 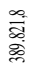 & 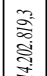 & $\stackrel{8}{8}$ \\
\hline & & 홍 존 & 总 & $\begin{array}{l}\text { 䄈 } \\
\equiv\end{array}$ & 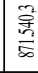 & ప & 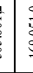 & है. & 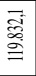 & 送 & 芳 & 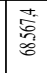 & 惫 & 言 & 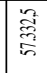 & 竞 & 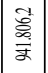 & $\begin{array}{c}\overrightarrow{\mathrm{o}} \\
\infty \\
\infty\end{array}$ & 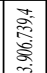 & $\frac{2}{2}$ \\
\hline & & 兰 & 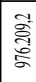 & $\begin{array}{l}\text { 晏 } \\
\text { 要 }\end{array}$ & 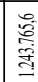 & 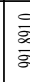 & & 8 & $\begin{array}{l}\text { 是 } \\
\text { 䞡 } \\
\end{array}$ & ๙ั. & 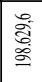 & 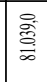 & 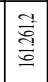 & 㮦 & 美 & ב్ & 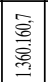 & है & 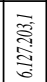 & $\frac{0}{2}$ \\
\hline & 萢 & 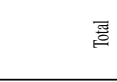 & 亲 & 器 & 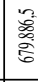 & $\bar{F}$ & 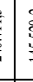 & 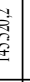 & 竞 & 誉 & 蛋 & 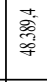 & 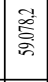 & $\begin{array}{l}\text { F- } \\
\text { 索 }\end{array}$ & 售 & 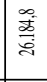 & \begin{tabular}{|l|}
$\stackrel{\circ}{\circ}$ \\
$\stackrel{0}{\infty}$ \\
$\stackrel{\equiv}{\rightleftharpoons}$
\end{tabular} & 哭 & 产 & 意 \\
\hline & 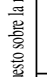 & $\cong$ & 胥 & 芯 & \begin{tabular}{|l}
$\frac{7}{3}$ \\
$\frac{3}{2}$ \\
$\frac{3}{2}$
\end{tabular} & & 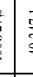 & F & हे & 告 & 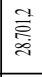 & $\begin{array}{l}\infty \\
\infty \\
\infty \\
\underline{\infty}\end{array}$ & 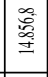 & 唁 & 胥 & 总 & 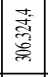 & 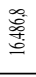 & 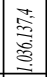 & 些 \\
\hline & & 20 & $\begin{array}{l}\infty \\
\text { aี } \\
\text { aี }\end{array}$ & $\bar{g}$ & \begin{tabular}{|l} 
音 \\
辛
\end{tabular} & $\Xi$ & 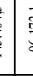 & 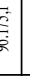 & 今ે & 总 & \begin{tabular}{|l|}
$\vec{\infty}$ \\
0 \\
0 \\
0
\end{tabular} & 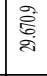 & 志 & 無 & $\mid$ & 発 & 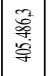 & 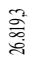 & 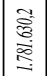 & $\frac{\mathrm{s}}{\mathrm{s}}$ \\
\hline & & 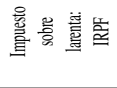 & 章 & స్లై & 嘀 & & & & 售 & $\mid$ & 死 & 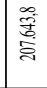 & 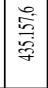 & 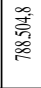 & 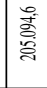 & 签 & 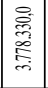 & 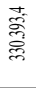 & $\begin{array}{l}\text { 월 } \\
\text { ปे } \\
\text { : }\end{array}$ & so \\
\hline & & & 是 & & 彎 & & & & 管 & 量案 & 唈 & 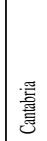 & 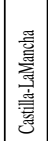 & 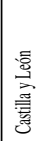 & 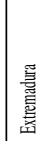 & 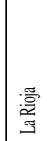 & 器 & 嘿 & छ & : \\
\hline
\end{tabular}




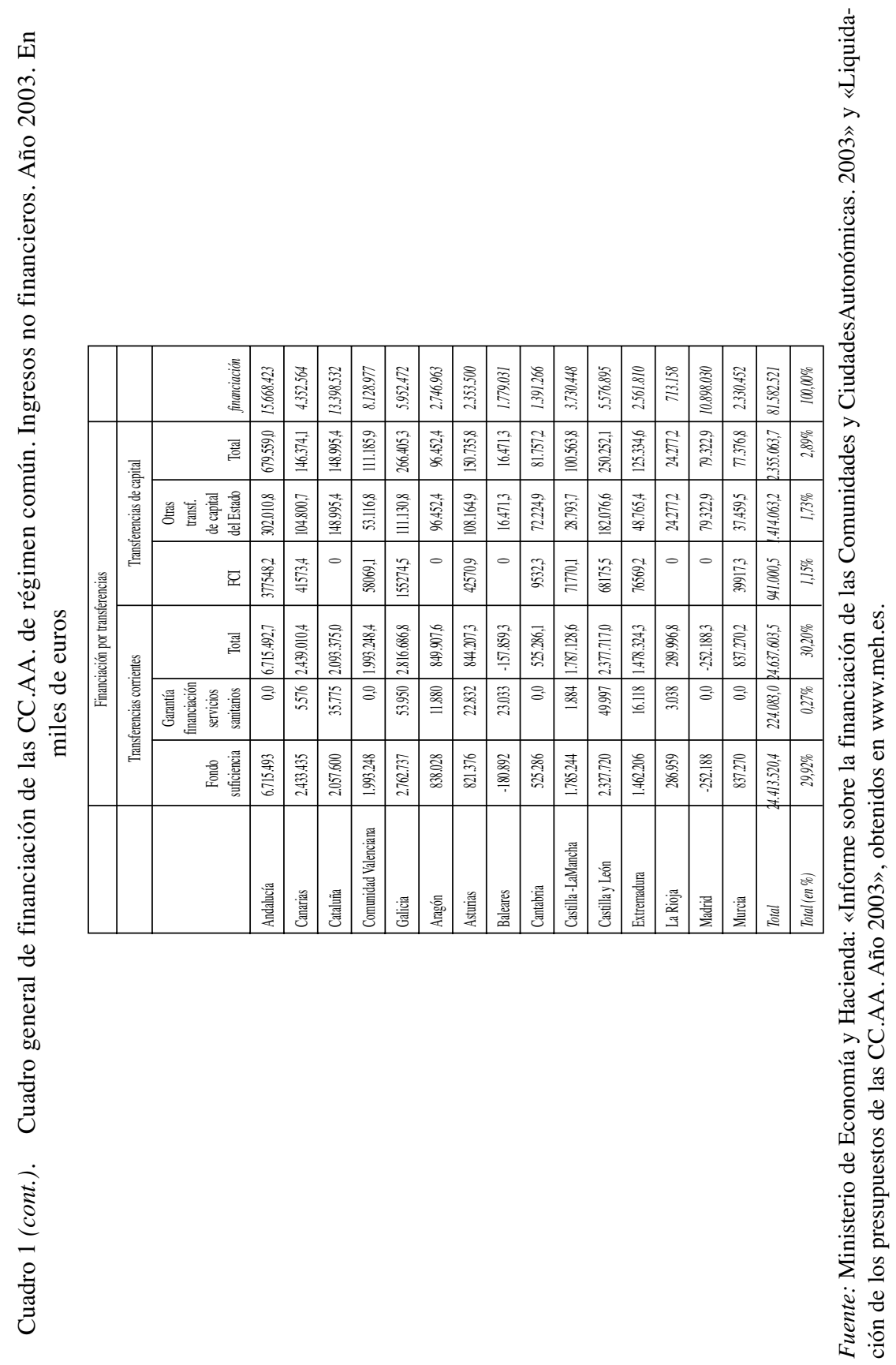




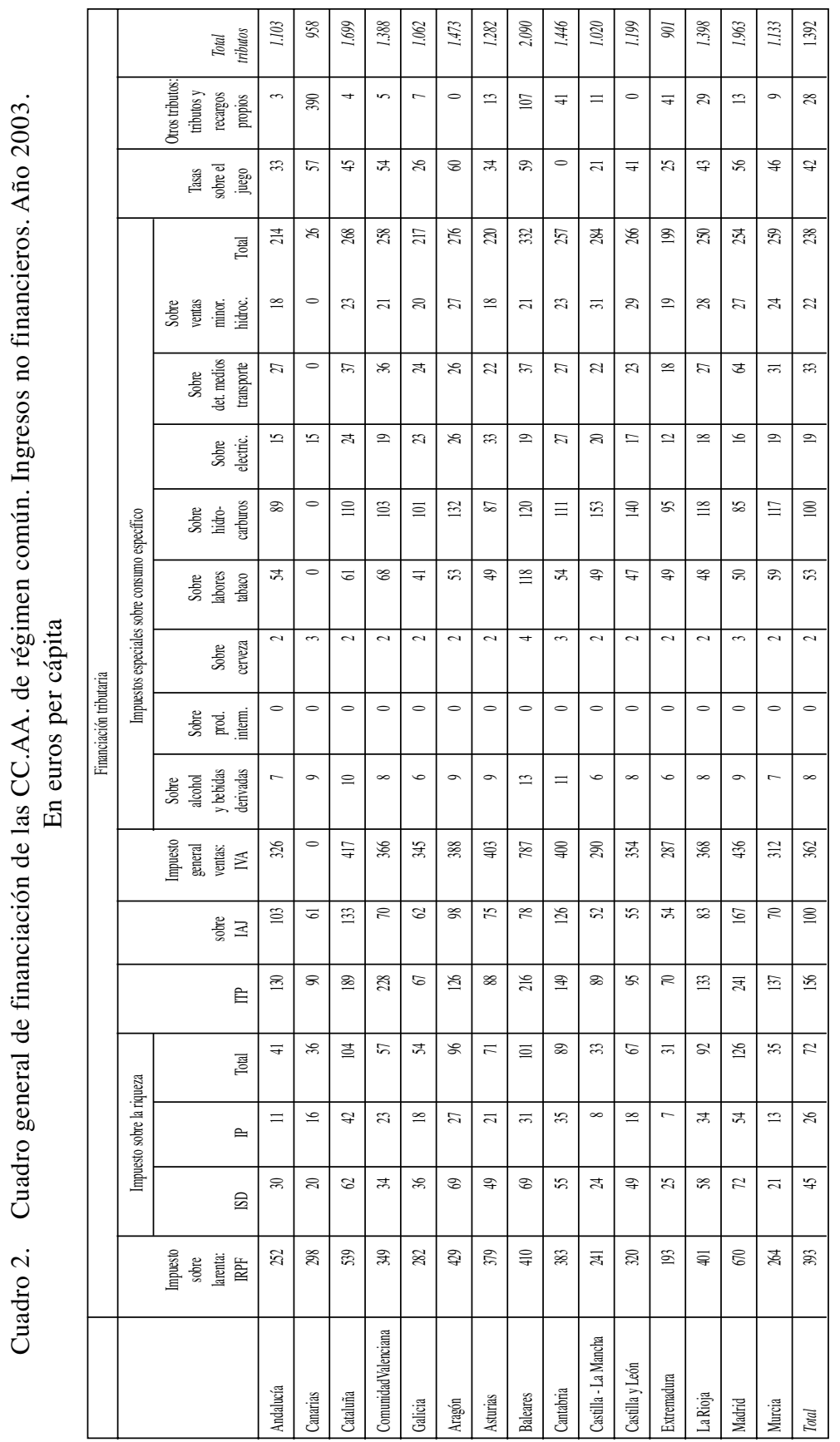




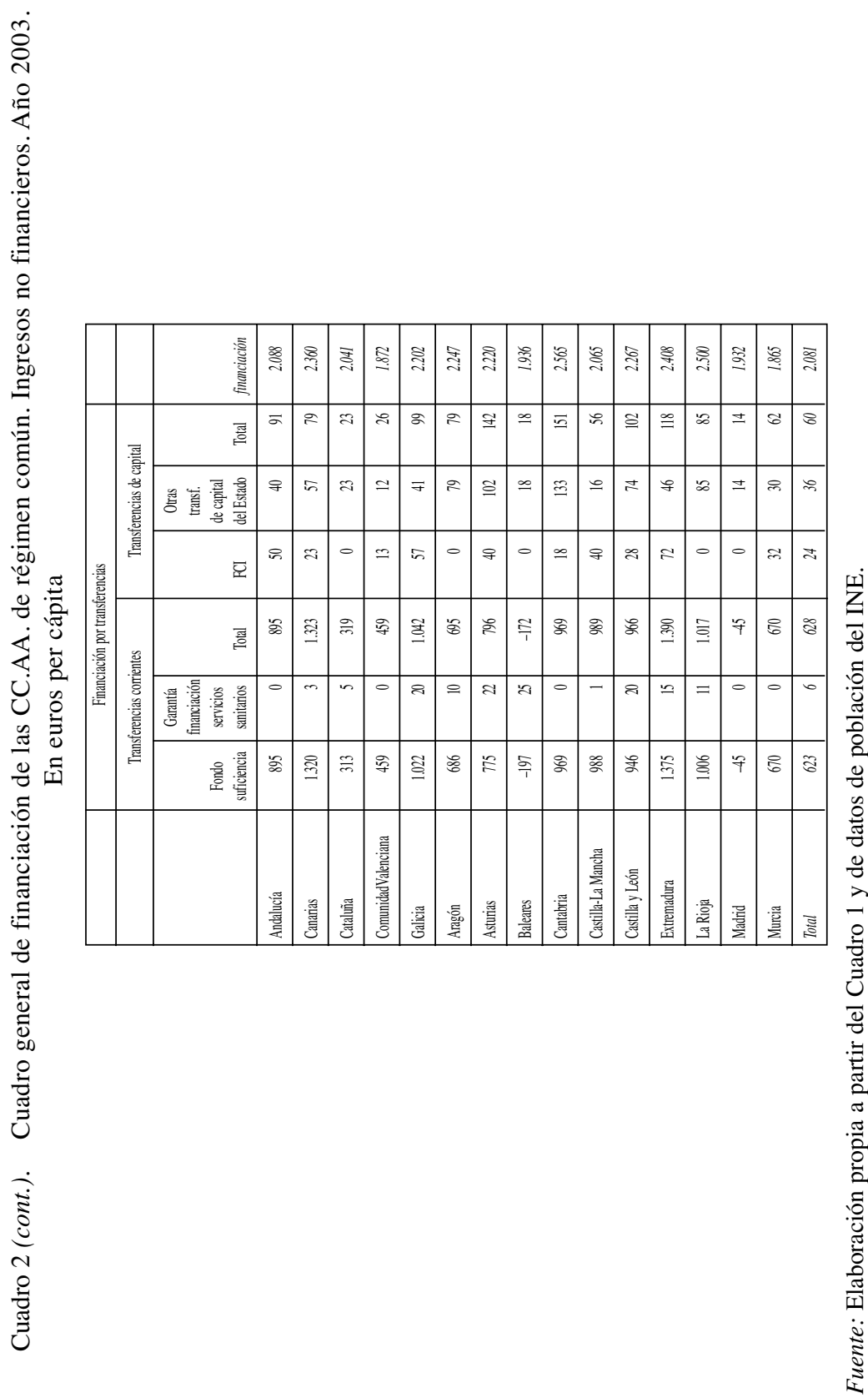


Pues bien, haciendo un breve resumen de los resultados obtenidos se aprecia que la distribución de recursos para el año 2003 presenta una desigualdad relativamente baja, representada por el índice de concentración del 0,0426, mientras que los tributos presentan un índice de 0,1413 y las transferencias uno del 0,3734. Por lo que se refiere a los índices representativos de la desigualdad en la distribución de cada recurso específico éstos presentan en su mayoría las características esperadas. Así los recursos procedentes de los impuestos sobre la riqueza se distribuyen más desigualmente que los que provienen del IRPF y estos a su vez más que los que se obtienen gravando el consumo.

No obstante la mayor desigualdad aparece en la distribución de los ingresos procedentes de las fuentes que implican el ejercicio de la capacidad tributaria propia, los denominados Tributos y Recargos Propios, concepto que engloba a un conjunto muy heterogéneo de tributos y recargos en el que se encuentran tanto los ingresos heredados de las Diputaciones Provinciales en el caso de las CC.AA. uniprovinciales, como los derivados del régimen tributario especial de Canarias, como tributos de nueva creación establecidos con carácter singular en algunas CC.AA. En el Cuadro 3 se puede observar que su índice de desigualdad es el más alto, siendo causas relevantes de esta desigualdad los casos de Canarias y Baleares. Baleares porque ese año aplicaba el impuesto sobre estancias en empresas turísticas de alojamiento (la denominada «ecotasa»), cuya recaudación alcanzaba 53,6 millones de euros, es decir, el 54,4\% del total de tributos propios de esta C.A.; y Canarias porque es titular de impuestos como el de los combustibles derivados del petróleo, el Impuesto general indirecto canario y los Recursos del Régimen Económico y Fiscal canario ${ }^{8}$. Esto último también afecta a los índices de los recursos derivados de IVA y a los Impuestos Especiales, puesto que si eliminamos el efecto distorsionador de Canarias, simulando el cálculo del índice de concentración sin esta C.A., por ejemplo en el IVA, el índice resultante es de 0,0934, índice más acorde con los que se pueden observar en el Cuadro 3 respecto al conjunto de Impuestos Especiales y respecto a aquellos Impuestos Especiales donde sí participa Canarias.

Por su parte, en cuanto a las transferencias, todas ellas presentan índices de desigualdad muy superiores a los de los impuestos, excluidos los Tributos y Recargos

${ }^{8} \mathrm{Si}$ simulamos el cálculo del índice de desigualdad de los tributos y recargos propios sin computar a Canarias, el índice disminuiría a 0,5741. Si eliminamos a Canarias y Baleares, el índice llegaría a 0,4789. 


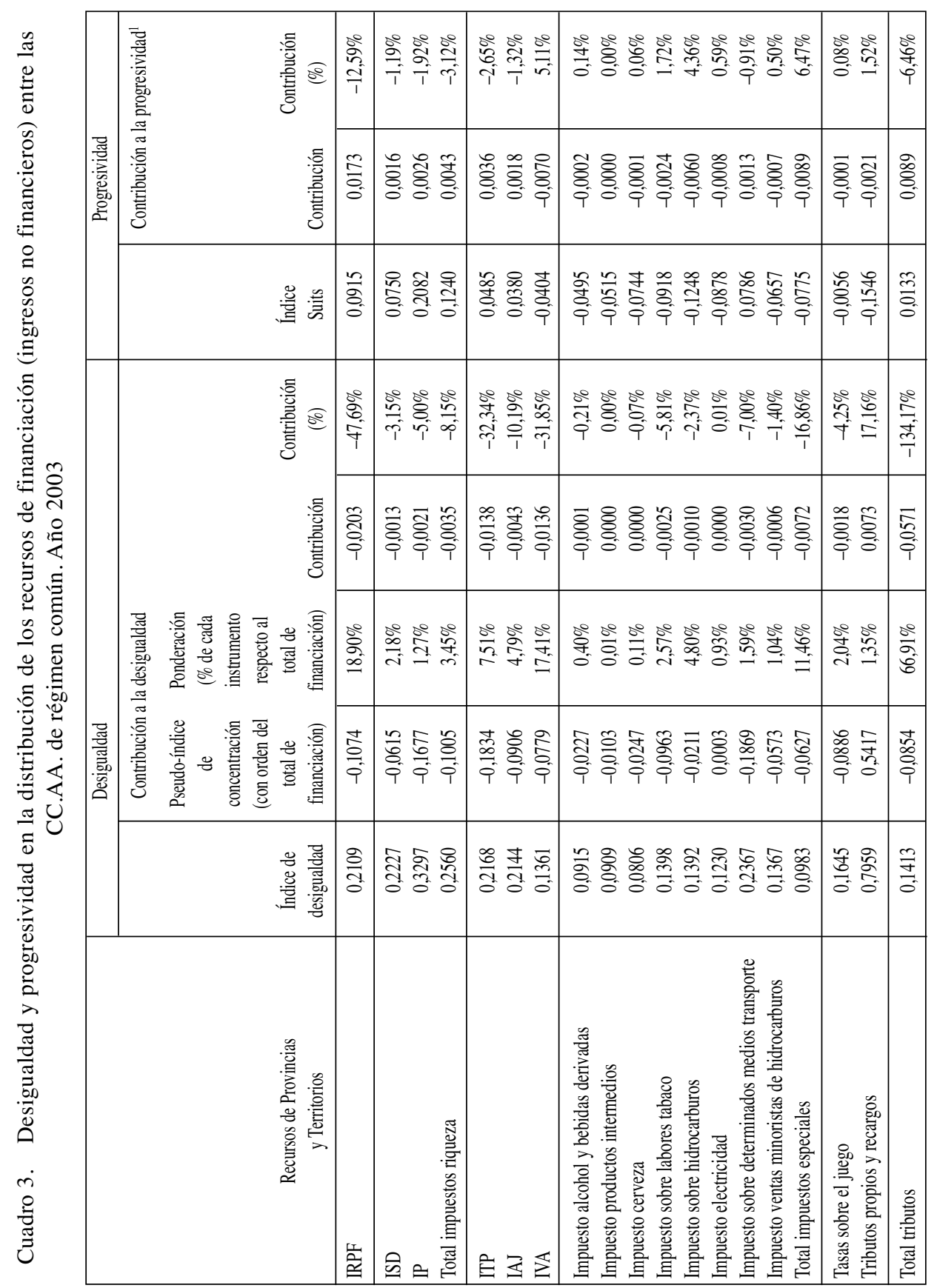


EL ORIGEN DE LA DESIGUALDAD Y LA PROGRESIVIDAD EN LA DISTRIBUCIÓN DE LA FINANCIACIÓN DE LAS COMUNIDADES AUTÓNOMAS DE RÉGIMEN COMÚN

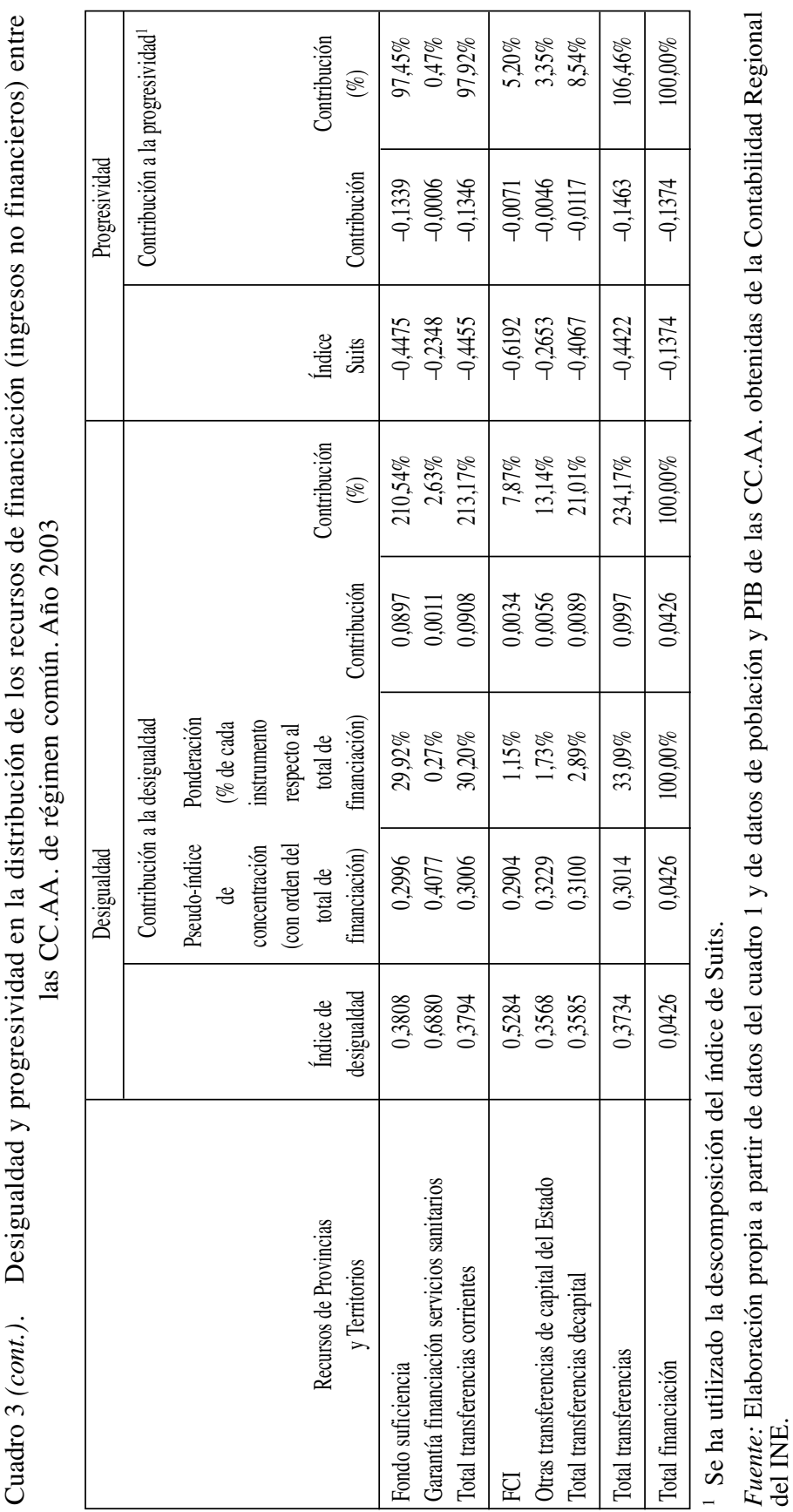


Propios. Los recursos procedentes de la transferencia de garantía de financiación de los servicios sanitarios y los procedentes del Fondo de Compensación Interterritorial son los que presentan mayor desigualdad, en ambos casos como consecuencia de que no todas las CC.AA. obtienen ingresos por esta vía. Como se puede observar en el Cuadro 2 para ambos casos el reparto es muy desigual.

La desigualdad implícita en cada instrumento de financiación nos da una idea de la distribución de cada fuente de ingresos pero, como ya se señaló, no aporta información en relación a la contribución a la desigualdad de distribución de los recursos totales. De hecho si sumamos ponderadamente las desigualdades observadas de impuestos y transferencias, obtendríamos un valor de 0,2181 , que tal como se predice en la ecuación 1 es muy superior a la desigualdad en la distribución de ingresos totales que se sitúa en 0,0426 .

Aplicando, tal como se propone más arriba, la descomposición natural de Shorrocks, es decir utilizando pseudos-índices de concentración, si sumamos la contribución de tributos y transferencias que aparece calculada en la columna 4 del Cuadro 3 se cumple la igualdad. Los tributos aportan una desigualdad de -0,0571 y las transferencias 0,0997, lo que significa, siguiendo la explicación hecha al comienzo del epígrafe, que mientras la recaudación por tributos, al presentar un signo negativo, pro-

Gráfico 1. Pseudo-Curvas de concentración de los recursos financieros de las Comunidades Autónomas de régimen común. Año 2003

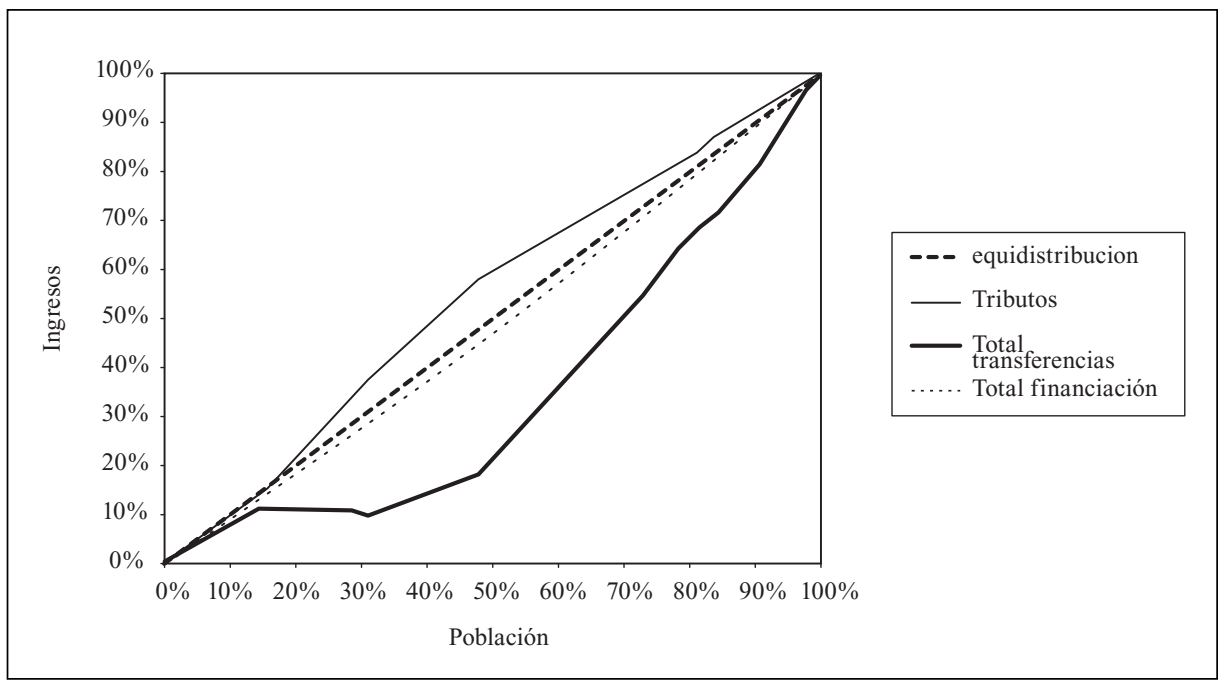

Fuente: Elaboración propia a partir de Cuadros 1 y 2 y de datos de población del INE. 
duce una desigualdad que opera en contra de la desigualdad final, los ingresos por transferencias producen una desigualdad en el mismo sentido que la del conjunto de recursos. En el Gráfico 1, donde se han representado la pseudo-curvas de concentración, se puede observar claramente cómo mientras las pseudo-curvas de concentración de los ingresos totales y de las transferencias están por de bajo de la línea de isodistribución, la correspondiente a los ingresos por impuestos está por encima.

El valor negativo que se da para los ingresos procedentes del conjunto de los tributos se reproduce en la práctica totalidad de los impuestos, destacando los casos de los ingresos procedentes de los impuestos sobre determinados medios de transporte, sobre transmisiones patrimoniales, sobre el patrimonio y sobre la renta, donde en todos ellos se obtienen pseudos-índices de concentración inferiores a - 0,1 .

En realidad, el sentido de la distribución final de recursos lo marcan los recursos procedentes del Fondo de Suficiencia, lo cual viene explicado por su función de mecanismo de cierre del sistema de financiación. En efecto, el modelo vigente a partir de 2002 fija una restricción financiera inicial para el año base 1999 compuesta por las liquidaciones para ese año de los recursos financieros del anterior modelo y formada por tres bloques de financiación: las competencias comunes, los servicios sanitarios y los servicios sociales. En cuanto a las competencias comunes, su Fondo General está compuesto por las liquidaciones para el año 1999 de la tarifa autonómica del IRPF, la PIE, tanto en su tramo general como en su tramo de participación en el IRPF, el Fondo de Garantía y la recaudación normativa por tributos cedidos y tasas afectas a servicios traspasados. En cuanto al bloque de las competencias sanitarias y sociales, están compuestos por las liquidaciones respectivas de su financiación para dicho año. Una vez cuantificadas dichas restricciones iniciales de financiación de los tres bloques mencionados, su reparto se efectúa, a grandes rasgos, de la siguiente manera. En cuanto al bloque de financiación de las competencias comunes, los indicadores de reparto son: la población (con una ponderación del 94\%), la superficie $(4,2 \%)$, la dispersión $(1,2 \%)$ y la insularidad $(0,6 \%)$. En cuanto al bloque de los servicios sanitarios, se distribuye según los siguientes indicadores: población protegida (ponderación del 75\%), población mayor de 65 años $(24,5 \%)$ e insularidad (0,5\%); en cuanto a los servicios sociales su reparto se efectúa según la población mayor de 65 años. A las cantidades correspondientes a cada C.A. se le deduce la recaudación normativa de los tributos cedidos y el resultado es su dotación por Fondo de Suficiencia. En consecuencia, esta dotación está concebida para compensar la desigual distribución de los recursos tributarios del nuevo modelo y para que la financiación autonómica tienda a una nivelación de las necesidades de gasto que orienta la desigualdad en sentido inverso al de la recaudación tributaria. 
Esto último nos traslada a la medición de la progresividad. Si tomamos las cifras del índice de Suits se aprecia que la progresividad del conjunto de la financiación es fruto del reparto del Fondo de Suficiencia, que contribuye en casi un $97,5 \%$ a la misma. Los ingresos por IVA y por impuestos especiales corrigen los resultados regresivos de los procedentes de impuestos directos y sobre la transmisión del patrimonio, y la parte de regresividad que no es compensada por éstos se compensa con el resultado progresivo del reparto del FCI y de las restantes transferencias de capital.

Esta situación queda claramente reflejada en el Gráfico 2, donde hemos representado la curvas de concentración relativa (de Suits) y en el que se puede observar que para los ingresos por los tributos, partiendo de una pauta de casi perfecta proporcionalidad en el reparto que se concentra entre las siete CC.AA. más pobres (Extremadura, Andalucía, Castilla-La Mancha, Galicia, Murcia, Asturias y Castilla-León), se produce un «salto» en el punto en que se acumula Canarias que hace que a partir de ahí la curva se sitúe muy ligeramente por debajo de la línea de equidistribución, indicando, por tanto esa leve regresividad mencionada anteriormente. Mientras que para los ingresos por transferencias tienen una distribución claramente progresiva. El hecho de que la curva de Suits para los ingresos totales sea menos distante a la de los impuestos que a la de las transferencias se debe a que el peso de los ingresos por tri-

Gráfico 2. Curva de concentración relativa de los recursos financieros de las Comunidades Autónomas de régimen común. Año 2003

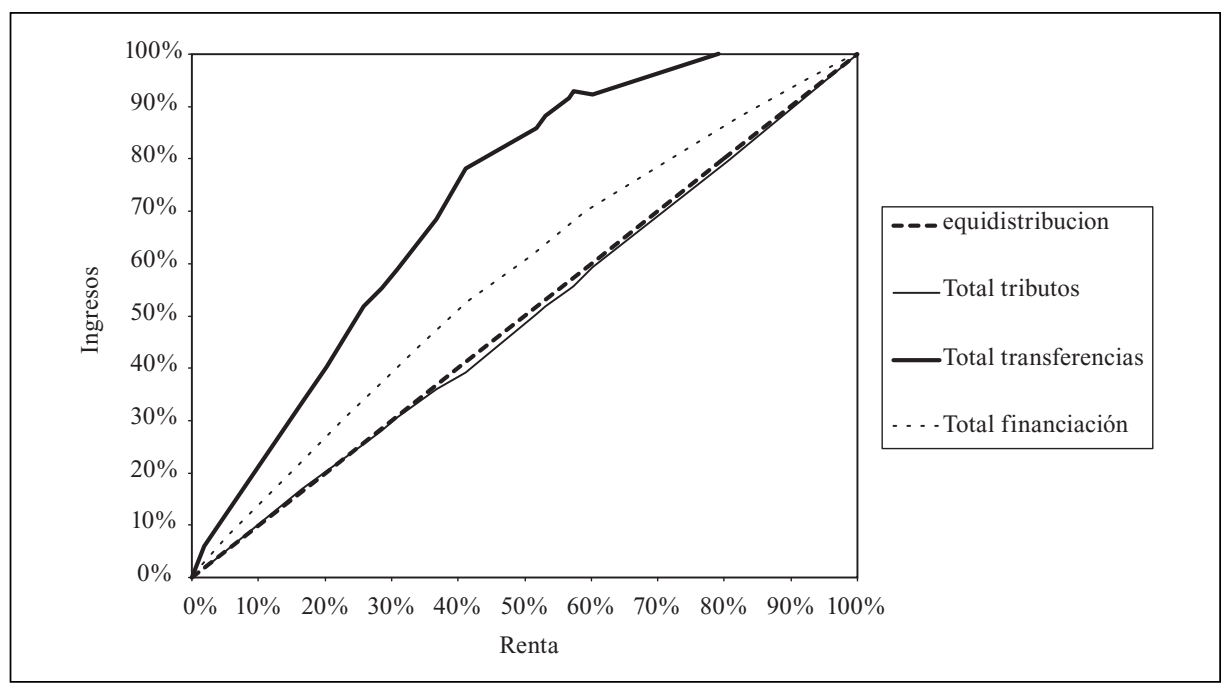

Fuente: Elaboración propia a partir de Cuadros 1 y 2 y de datos de población del INE. 
butos en el conjunto de la financiación es el doble al de los ingresos por transferencias.

\section{Conclusiones}

Como se puede observar, la metodología propuesta supone un importante recurso de información sobre el sistema de financiación. La metodología utilizada ha consistido en trasladar la descomposición aditiva del índice de concentración, algo muy común en otros ámbitos de estudio de la economía, a la financiación autonómica. Además, aprovechando la singularidad del índice de Suits para su descomposición hemos utilizado la misma para poder calificar el sentido de la distribución de cada fuente de ingresos en progresiva o regresiva y conocer su aporte al sentido de la distribución final. Como se ha señalado más arriba, esta última descomposición tiene menos problemas de interpretación en la medida en que todos los instrumentos de financiación mantienen una misma ordenación de las CC.AA. en el cálculo del índice de Suits, lo que no ocurre en el caso de la descomposición del índice de concentración, lo que obliga al cálculo de pseudo-índices más difíciles de interpretar. Con todo, la metodología resulta interesante ya que permite calcular la contribución de cada instrumento de financiación a la desigualdad y progresividad globales del sistema, y con ello esclarecer el origen de las mismas, objetivo básico de este trabajo. Ello supone una mejora en la medición de la distribución de los recursos en los sistemas de financiación federales.

En cuanto a las conclusiones derivadas de la aplicación de dicha metodología al caso español es evidente que los resultados distributivos finales del sistema autonómico de financiación vienen determinados por el método de cálculo del Fondo de Suficiencia y su aplicación como variable de cierre del modelo de financiación, lo cual hace perfectamente previsible que sus criterios de reparto determinen la desigualdad final en la distribución de recursos y su sentido progresivo. No obstante es importante conocer la repercusión que tienen las demás transferencias y en especial los ingresos por transferencias de capital, FCI y otras transferencias, para mantener los resultados distributivos. De hecho si se observan las cifras se aprecia que, si bien las transferencias de capital no suponen un aporte relevante en desigualdad, o sea no son desequilibradoras, ya que su contribución no llega ni a 0,009 para un índice de desigualdad de 0,0426, sin embargo son fundamentales para compensar la pérdida de progresividad que deriva de los impuestos directos y de Transmisiones Patrimoniales y Actos Jurídicos Documentados en el sistema. Esa misma función cumplen los ingresos por 
impuestos indirectos, IVA y especiales que entre ambos corrigen casi la regresividad del IRPF, lo cual está muy relacionado con la discusión sobre la cesta de tributos que se planteó allá por la segunda mitad de los noventa cuando se aumentó el peso del IRPF en la financiación. No obstante, en este último caso la importancia hay que relativizarla, ya que de no haberse ampliado la cesta de tributos la consecuencia inmediata habría sido un crecimiento de la progresividad del Fondo de Suficiencia por ser la fuente que cierra el sistema.

En definitiva lo más relevante es que las transferencias de capital y en particular el FCI, que no está sujeto a la discrecionalidad del Gobierno, son elementos importantes para evitar que finalmente pierda parte de la progresividad derivada de la variables distributivas del Fondo de Suficiencia.

\section{REFERENCIAS BIBLIOGRÁFICAS}

Aronson, J.R., Johnson, P., Lambert P.J. (1994): «Redistributive effect and unequal income tax treatment», The Economic Journal, vol. 104, núm. 423, págs. 262-270.

BIRD, R., TARASOv A.V. (2002), «Closing the gap: fiscal imbalances and intergovernmental transfers in developed federations», Working Paper 02-02, Andrew Young School of policy studies, Georgia State University.

BoAdwAy, R. (2001), «Intergovernmental fiscal relations: the facilitator of fiscal decentralization», Constitutional Political Economy, Vol. 12, núm. 2, págs. 93-121.

- (2003), «The theory and practice of equalization», WP 1016, Department of Economics, Queen «s University.

Brodjonegoro, B., Martínez-Vázquez, J. (2002), «An analysis of Indonesia«s transfer system: recent perfomance and future prospects» Working Paper 02-13, Andrew Young School of policy studies, Georgia State University.

Buchanan, J.M. (1950), «Federalism and fiscal equity», American Economic Review, vol. 40, núm. 4, págs. 583-99.

KING, D.N. (1984), Fiscal Tiers: The economics of multi-level government, George Allen and Unwin, Londres.

FeI, J.C.H., Ranis, G., Kuo, S.W.Y. (1978) : «Growth and the family distribution of income by factor components», The Quarterly Journal of economics, vol. 92, núm. 1, págs. 1753.

FISHER, R.C. (ed.) (1997), Intergovernmental fiscal relations, Kluwer Academic Publishers, Boston.

GoerLICH, F.J. (1998), «Desigualdad, diversidad y convergencia: (algunos instrumentos de medida)», Instituto Valenciano de Investigaciones Económicas, www.ivie.es/downloads/docs/mono/mono1998-01.pdf (consulta 23-4-2008).

Hierro, L.A., Atienza, P., PatiÑo, D. (2007a), «Inequality and progressiveness in the distri- 
bution of revenues of the states in federal countries. A comparative study», Working Paper 07-03, International Studies Program, Andrew Young School of policy studies, Georgia State University.

Hierro, L.A., Atienza, P., Patiño, D. (2007b), «Un análisis de la distribución de las transferencias intergubernamentales en Australia», Revista de Estudios Regionales, núm. 78, págs. 307-316.

Hofman, B., Cordeira Guerra, S. (2004), «Ensuring interregional equity and poverty reduction», Working Paper 04-11, Andrew Young School of policy studies, Georgia State University.

Kakwani, N.C. (1977): «Measurement of Tax Progresivity: An International Comparison», Economic Journal, núm. 87 (345), págs. 71-80.

LAdD, H.F., YINGER, J. (1994), «The case for equalizing aid», National Tax Journal, vol. 47, núm. 1, págs. 211-24.

Lerman, R.I., YITZHAKI, S. (1985): «Income inequality by income source: a new approach and applications to the United States», Review of Economics and Statistics, LXVII(1), págs. 151-156.

MacDougall, D. (1977), Report of the Study Group on the role of public finance in European integration, Commission of the European Communities, Bruselas.

Martínez-Vázquez, J., Boex, J. (1999), «Fiscal decentralization in the russian federation during the transition», Working Paper 99-3, Andrew Young School of policy studies, Georgia State University.

- (2001), «The design of equalization grants: theory and applications», World Bank Institute, Andrew Young School of policy studies, Georgia State University.

Mathews, R. L. (1977), «Mechanisms for Fiscal Equalisation in an integrating economic community», en MacDougall, D. (1977).

Mieszkowski, P.M., Musgrave, R.A. (1999), «Federalism, grants, and fiscal equity», National Tax Journal, núm. 52, págs. 239-260.

Musgrave, R. A. (1961), «Approaches to a fiscal theory of political federalism», en N.B.E.R. (Ed.) (1961), págs. 97-122

N.B.E.R. (Ed.) (1961), Public Finances: Needs, sources and utilization, Princeton University Press, Princeton.

OAKLAND, W.H. (1994), «Fiscal equity, an empty box?», National Tax Journal, vol. 47, núm. 1, págs. 199-210.

OAtes, W. E. (1972), Fiscal Federalism, Harcourt Brace Jovanovich, N.Y.

Petchey, J., Shapiro, P, Walsh, C. (1997), «Transfers in federal systems: a critical survey», capítulo 4 de Fisher (1997).

Rao, M.G. (2000), Fiscal decentralization in Indian federalism, International Monetary Fund, Washington.

RAo, V. M. (1969), «Two decompositions of concentration ratio», Journal of the Royal Statistical Society. Series A, vol. 132, núm. 3, págs. 418-425.

Sнна, A. (1983), «Efficiency, equity and fiscal equalization grants: issues and alternatives», Finance Canada, Discussion Paper. 
Shorrocks, A. F. (1982), «Inequality decomposition by factor components», Econometrica, vol. 50, núm. 1, págs. 193-212.

- (1999), «Decomposition procedures for distributional analysis: A unified framework based on the shapley value», Department of Economics, University of Essex, www.komkon.org/ tacik/science/shapley.pdf (consulta de 23-4-2008)

Surts, D. B. (1977), «Measurement of Tax Progressivity», American Economic Review, vol. 67(4), págs. 747-752.

Ter-Minassian, T. (ed.) (1997), Fiscal federalism in theory and practice, International Monetary Fund, Washington. 